\title{
A QGIS Tool for Automatically Identifying Asbestos Roofing
}

\author{
Maurizio Tommasini ${ }^{1}\left[\right.$, Alessandro Bacciottini ${ }^{1}$ and Monica Gherardelli ${ }^{2, *} \mathbb{C}$ \\ 1 PIN-Polo Universitario Città di Prato, Piazza Giovanni Ciardi 25, 59100 Prato, Italy; \\ maurizio.tommasini@gmail.com (M.T.); alessandro.bacciottini@gmail.com (A.B.) \\ 2 Department of Information Engineering, University of Florence, Via di Santa Marta 3, 50139 Florence, Italy \\ * Correspondence: monica.gherardelli@unifi.it
}

Received: 25 January 2019; Accepted: 24 February 2019; Published: 6 March 2019

\begin{abstract}
Exposure to asbestos fibers implies a long-term risk for human health; therefore, the development of information systems that are able to detect the extent and status of asbestos over a certain territory has become a priority. This work presents a tool (based on the geographic information system open source software, QGIS) that is conceived for automatically identifying buildings with asbestos roofing. The area under investigation is the metropolitan area around Prato (Italy). The performance analysis of this system was carried out by classifying images that were acquired by the WorldView-3 sensor. These images are available at a low cost when compared with those obtained by means of aerial surveys, and they provide adequate resolution levels for roofing classification. The tool, a QGIS plugin, has shown fairly good performance in identifying asbestos roofing, with some false negatives and some false positives when applying a per-pixel classification. A performance improvement is obtainable when considering the percentage of asbestos pixels that are contained in each roof of the analyzed image. This value is also available with the plugin. In the future, this tool should make it possible to monitor the asbestos roof removal process over time in the area of interest, in accordance with other image data that give evidence of such removals.
\end{abstract}

Keywords: asbestos identification; image analysis; open source geographic information system; remote sensing

\section{Introduction}

Between the 1970s and the 1990s, asbestos was widely used in buildings for its particular characteristics of resistance and its good insulating properties. Specifically, flat sheets and cement boards containing asbestos were used as building materials in roofing, both in industrial and civilian infrastructures. Some years after their installation, such plates tended to release a huge amount of fibers, particles, and fractions of asbestos that were of inhalable size. The main problem lies in the fact that asbestos fibers tend to divide lengthways into thinner fibrils that are small enough to penetrate the lung alveoli, thus causing very serious diseases [1,2].

Despite this danger, and regardless of the ban on using asbestos in any business context, a prohibition that is carried out in many countries, there are still many buildings with asbestos roofs. In Italy, many old buildings still have Eternit cladding (asbestos fibers reinforced with cement material), even though the national law (no. 257 of March 1992) forbids the use of asbestos.

Most people are not aware of the danger, and thus, they may not properly dispose of it during roof renovations. Accordingly, a thorough and exact list of the roofs posing a risk in terms of asbestos pollution is required [3].

Therefore, one priority is to set up and to finalize information systems that are capable of describing the extent and conditions of asbestos-related materials in a given area. 
There are different approaches that can be adopted for this reconnaissance. The use of remotely sensed data has proven to be a good instrument for identifying and evaluating the status of roofing made of asbestos fiber cement materials, e.g., by using images from space-borne remote sensing [4-8]. Due to the spectral characteristics of asbestos roofs, hyperspectral remotely sensed images are often used for asbestos roofing identification [3,9-16].

It is worthwhile to observe that space-borne sensors allow for a larger coverage area, and they can be cheaper when compared with airborne sensors, such as the hyper-spectral sensor MIVIS (multispectral infrared visible imaging spectrometer) [17]. Obviously, the use of imagery from space-borne sensors means that the spatial and spectral resolution will be lower than with airborne sensors.

Recent papers illustrate the possibility of exploiting remotely sensed images from sensors on board the WorldView satellites. Pacifici shows that improvements in classification accuracy are obtainable by using the WorldView-3 spectral bands, as compared with the more typical platforms [17]. Some researchers used an object-based approach to extract information from the images and have proven that their processing methods have the potential to detect roof materials from the WorldView-2 images $[18,19]$. More recently, other authors have illustrated research carried out with discriminant function analysis (DFA) and random forest (RF) on WorldView-2 imagery [20]. Their aim was to reveal the efficiency of these classifiers, and the efficiency of pansharpening. The best results were gained by $\mathrm{RF}$, with both three and six classes. The results of the last paper confirm some choices that were made during our research.

This work arises from this context, and its goal is to provide a directory where all of the buildings with asbestos cladding can be listed for a given area. To meet this requirement, this paper proposes a low-cost tool that is designed to automatically identify buildings with asbestos roofing in a particular area. Its task is to identify asbestos cladding in remotely sensed images, but not to classify the different types of roofing. The tool is designed as a QGIS plugin, named 'RoofClassify'. QGIS is an open source geographic information system licensed under the GNU General Public License [21]. The version used in this work is QGIS 2.18. The same tool presented here should make it possible in the future to monitor the entire process of asbestos roofing removal in a selected area over time, and by means of subsequent digital imaging.

In the framework of this application, the considered area is the large metropolitan area around Prato, which is the second most populated city in Tuscany (Italy). Images acquired by the WorldView-3 sensor were selected and processed. It is known that classifying these images is a difficult task, because urban areas are characterized by different surface features that have similar spectral responses [22]. The probability of incorrectly classifying these surfaces thus increases. Traditional pixel-based classifications seem unsuitable, whereas object-oriented classifications are more advisable $[18,19,23]$.

The RoofClassify plugin uses data integration techniques (vector and raster) for representing WorldView-3 data onto a common grid. It then selects building-objects using vector format features, and it finally applies a classification procedure to the roofing of buildings. The result of this process is a new feature for each building-object. This feature is immediately usable within the integrated geographic information system (GIS) environment for the analysis of each point characteristic (vector and raster information), and for a statistical survey of the observed area.

This approach makes it possible to filter the images without using, or training, object-oriented classification algorithms or neural networks [20]. Moreover, it makes it possible to reduce errors due to false positives that are represented by objects like asphalt on roads. Finally, fast software packages can be used that are specifically designed for classifying pixel-based images.

Section 2 focuses on the justification of the choice of images classified. The same section presents the method used to detect Eternit cladding, and the designed open source software tool. The results obtained, and the explanations of the validations, are reported in Section 3. 


\section{Materials and Methods}

\subsection{Roofing Classification}

The roofing classification was performed through digital image processing within the selected area. The first step involved the kinds of images that the proposed software tool could be applied to.

\subsubsection{Image Selection and Preprocessing}

The following systems were among the different aerial images that were used to reproduce the Prato municipal district area:

- Orthophoto: aerial photographs or images that have been geometrically corrected and georeferenced ("orthorectified"), with radiometric resolution in four spectral bands (R,G,B, NIR) and with a spectral coverage of up to $\sim 800 \mathrm{~nm}$. Such images are available free of charge at the Geoscope Observatory in Tuscany (http:/ / www502.regione.toscana.it/geoscopio/ortofoto.html). Their low spectral resolution does not make it possible for their use in any asbestos classification.

- Vector graphics files, available at the Geoscope Observatory in Tuscany. Once the Geoscope website is accessed, the layer with the information requested can be chosen; for instance, surveyor maps.

- High spectral resolution images, acquired by airborne sensors (MIVIS) [14]. They are very expensive if there are specific acquisition procedures. Their broad representative potential is limited by the noise occurring in high-depth bands.

- Satellite images acquired by the WorldView-3 sensor working on eight spectral bands. WorldView satellite imagery is often more expensive than RGB aerial imagery if a comparison is made between the archives. In the presented case, no archive aerial imagery is available, and additionally, WorldView images of the area of interest can be purchased at fairly low prices (about 2200 euros) [24].

The need to have multispectral imaging and high spatial resolution [25] while trying to keep costs down, influenced the choices of the images to be used for this research, favoring those that were obtained by the WorldView-3 sensor. This sensor, owned by DigitalGlobe, is mounted on a commercial Earth observation satellite [24]. In August 2014, DigitalGlobe launched this multi-payload, super-spectral, very high spatial resolution satellite. Operating at an altitude of $620 \mathrm{~km}$, WorldView-3 acquires panchromatic imagery at a $0.31 \mathrm{~m}$ maximum spatial resolution, eight visible and near-infrared (VNIR) bands at $1.24 \mathrm{~m}$ maximum spatial resolution, and eight short-wave infrared (SWIR) bands at $3.7 \mathrm{~m}$ [17]. Since these images do not cover the asbestos emissions wavelength, it was possible to detect the fiber-cement roofing by analyzing the corresponding spectrometric signature, namely, by analyzing the typical reflection characteristics of this material to such wavelengths. Therefore, the classification process was adjusted accordingly, so as to fully exploit each and every piece of information that could be obtained from the available satellite images.

Figure 1 shows the steps of the classification process, organized in a block diagram. 


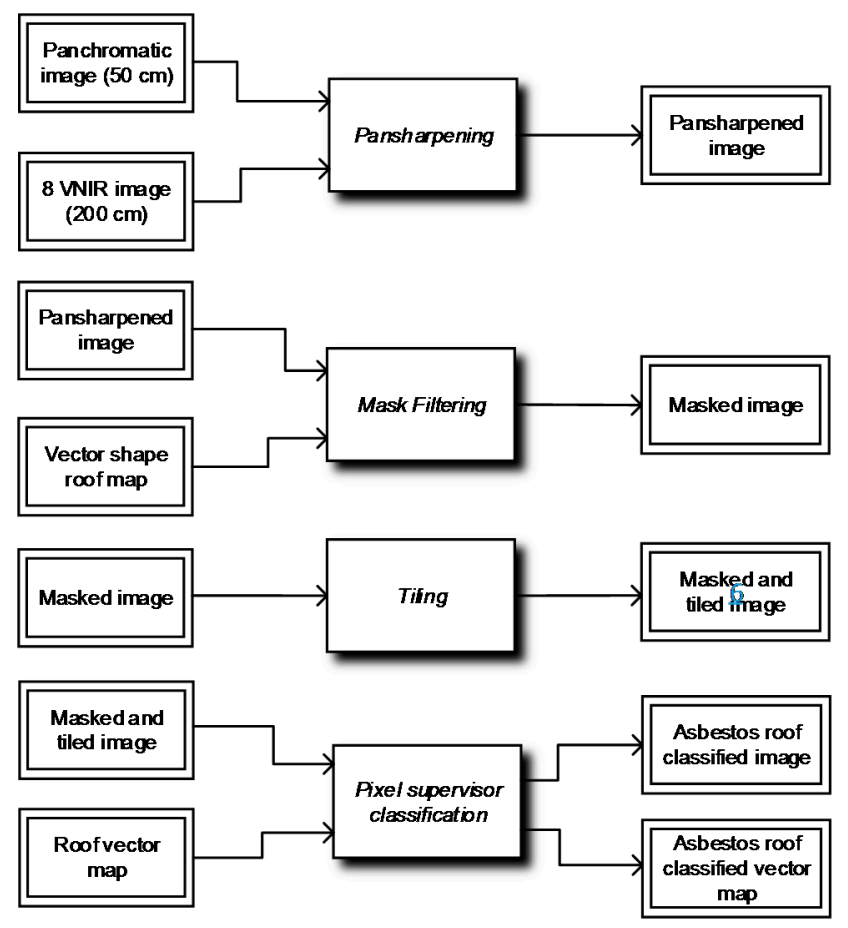

Figure 1. Illustration of the different processing steps of the classification process. VNIR is visible and near-infrared.

Two phases made up the process. The first non-automatic phase focused on preprocessing the available data, and it included three steps.

1. Pansharpening, the process that enabled a merger of the information collected from satellite imaging, as acquired by the WorldView- 3 sensor, in order to create a single image with the resolution of the initial panchromatic image (Figure 2). The low-resolution image was interpolated with bi-cubic interpolation. In this activity, pansharpening was carried out using a component substitution algorithm. The, RCS (relative component substitution) algorithm was selected among those available, through Orfeo Tool-box [26], a software integrated into QGIS. Table 1 describes the features of the images used for such processing:

- A panchromatic image with a higher spatial resolution $(50 \mathrm{~cm})$;

- A multispectral image having eight spectral bands (coastal, blue, green, yellow, red, red edge, NIR-1, NIR-2) and characterized by a spatial resolution of $2 \mathrm{~m}$.

These images were georeferenced in UTM33 WGS84 and orthorectified. They were also calibrated and corrected radiometrically. Our research started in 2016, and it aimed to document the diffusion of asbestos roofs in the Prato area in 2014. For this reason, the selected images were among those acquired in 2014. In fact, from that moment onwards, the authorities of Prato have updated their database by inputting the removal procedures of asbestos-contaminated artifacts by individuals and companies.

2. Raster image filtering, meaning filtering of the pixel grid, was obtained through a vector mask of the chosen area. The vector mask was something that ensued from the cadastral shape layer, namely, from the surveyor map where all of the buildings that were related to the selected area were described via a vector graphics editor (Figure 3). The inferred mask only contained information that related to the buildings' roofing. This process enabled the removal of everything but the buildings' roofing from the initial satellite image (Figure 4). The cropping operation was performed using the corresponding functions offered by QGIS and based on the Gdal libraries. 
3. Partition of the filtered image in tiles, namely sections that had suitable dimensions, so as to carry out the classification as quickly as possible. The partition of the satellite image was carried out with a plugin called GridSplitter, thus obtaining 49 sections, each of them correctly geo-referenced according to the projection of the original image. Such tiles were saved into a single folder containing only and exclusively these files.

Table 1. Characteristics of the satellite panchromatic image and of the multispectral image.

\begin{tabular}{|c|c|c|}
\hline Description & Panchromatic Image & Multispectral Image \\
\hline \multirow{2}{*}{ Image name } & ortho14nov02101210-p2as-055 & ortho14nov02101210-m2as-055 \\
\hline & 544307010_01_p001 & 544307010_01_p001 \\
\hline Source & Planetek Italia & Planetek Italia \\
\hline Size & $40785 \times 25825$ pixels & $10197 \times 6458$ pixels \\
\hline Imagine type & Pancromatic & Multispectral \\
\hline Number of bands & 1 & 8 \\
\hline Radiometric resolution (bit) & 16 & 16 \\
\hline Spatial resolution (cm) & 50 & 200 \\
\hline Acquisition date & $02 / 11 / 2014$ & $02 / 11 / 2014$ \\
\hline Reference system & Roma40/Ovest & Roma40/Ovest \\
\hline Resampling type & Bilinear Interpolation & Bilinear Interpolation \\
\hline Sensor & WorldView-3 & WorldView-3 \\
\hline Cloud cover $(\%)$ & 0 & 0 \\
\hline Off-nadir & $24.9^{\circ}$ & $24.9^{\circ}$ \\
\hline Metadata language & Italian & Italian \\
\hline Character set code & utf-8 & utf-8 \\
\hline
\end{tabular}

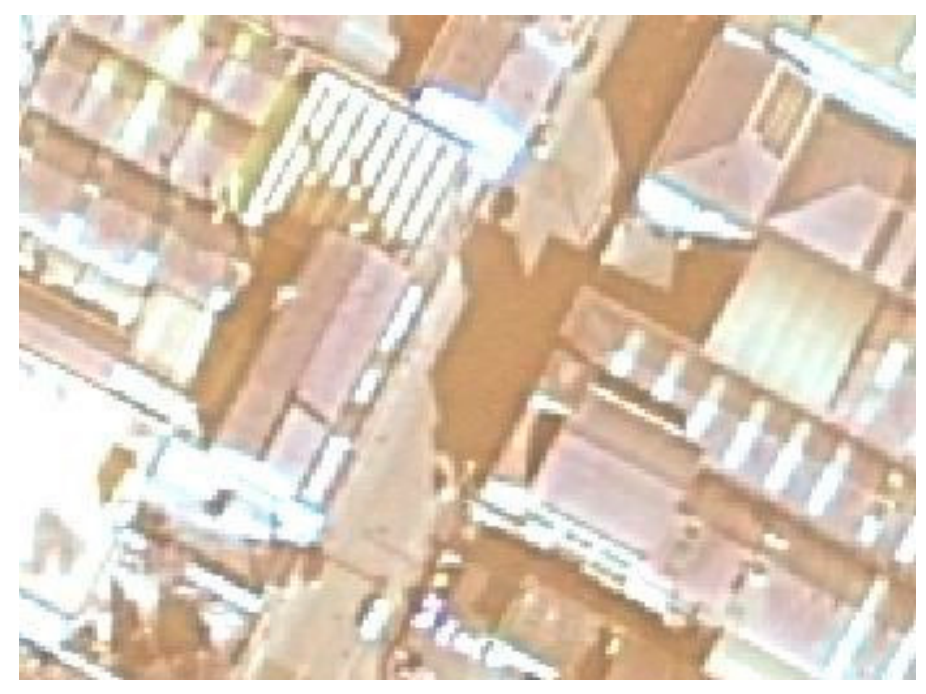

Figure 2. The multispectral image obtained via pansharpening (resolution: $50 \mathrm{~cm}$ ). 


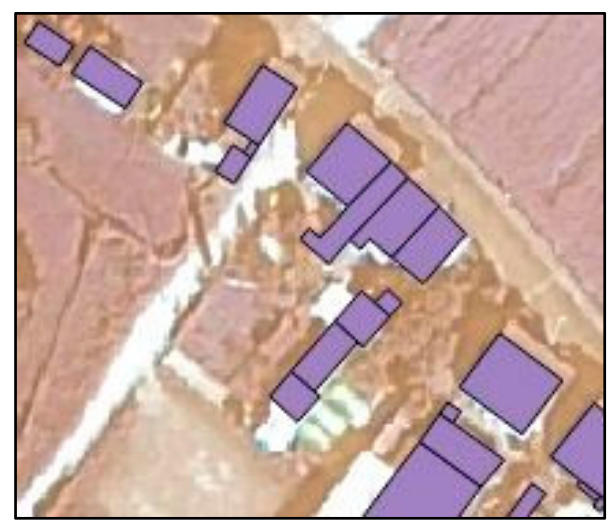

Figure 3. Overlapping of the shapefile mask related to buildings, using the pansharpened satellite image excerpt.

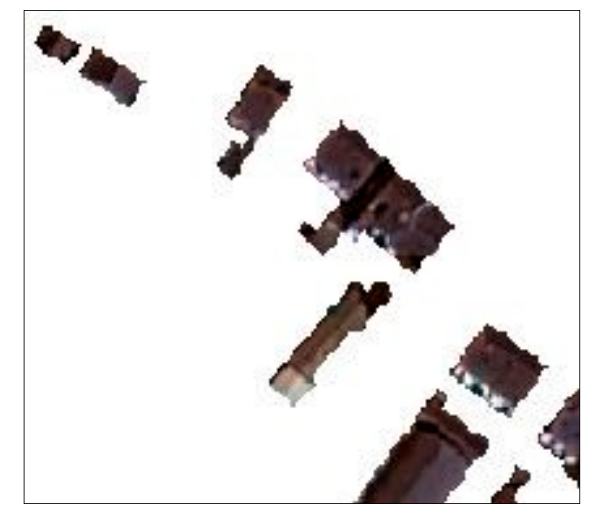

Figure 4. Mask filtering results related to the buildings involved.

\subsubsection{Classification Procedure}

The second phase was the real classification of the cladding available in the area according to the pre-processed image. Digital image classification techniques grouped pixels into classes, to represent the different types of land cover data. There were three main approaches in digital image classification: supervised, unsupervised, and object-oriented. The method applied hereafter was based on supervised classification, which operated a per-pixel classification of the image with some representative samples, the true points, for each and every land cover data class that appeared in the digital image. The choice of a supervised approach was not limiting, because it enabled the subsequent application of an object-oriented classification, if required, as shown further on.

In this case study, there were six selected classes: asbestos cladding, fiber cement cladding, $\mathrm{red} / \mathrm{red}$ roofing, black/shadow, green, and background. The last class collected pixels from objects that were different from the roofing. These pixels could be incorrectly located on a building roof due to incorrect processing of the image, such as inaccurate georeferencing. The white background pixels of Figure 4 also contributed to this class if the classification process also considered them.

The corresponding six training sets were composed of samples that were collected during a survey campaign carried out in the Prato area. At each site, the geographical position (latitude, longitude, WGS84 datum) of the surface was detected, and a link was associated with the vector record of the building, as provided by the land registry.

The image classification software therefore worked with the training sets to define the kinds of land cover in the entire image. Digital image classification determined the membership to each class according to the "resemblance" of the observed object's radiative properties to the properties defined in the training set. Among the different algorithms operating supervised classifications, the random forest algorithm was selected and applied to the available images. Indeed, this algorithm seemed 
to be more suitable, because as an ensemble of classifiers, it was capable of classifying each pixel by resorting to its characteristics, such as: its spectral properties in the eight different bands of the satellite sensor, its panchromatic value, and its membership to building class. Moreover, it worked well when homogeneous training data were available, and it was relatively robust to outliers. Last, but not least, implementation of this algorithm ensured a high computational speed [19,27-29]. Within this work, the classification process was totally automatic, and it was implemented with an algorithm that was specifically designed through a QGIS plugin.

\subsection{The RoofClassify Plugin}

The QGIS open source environment enables any data collection coming from different sources (raster, vector, database, and so forth), to be used to build a single project of spatial analysis that is capable of meeting the basic functionalities that a GIS has to cope with. Furthermore, a useful aspect of QGIS is the option of expanding basic functionalities through plugins in Python language, which is simple for any user to understand. This means that Python libraries that are related to both image management and processing can interact with the functionalities that a GIS has (for instance displaying an indexed map in some reference systems). This leads to the possibility of creating a simple application that efficiently copes with a particular problem: the application software has a user interface, and it works with both new and already existing application fields in QGIS, as defined by the user in Python language.

The RoofClassify plugin was developed within the application field of this current paper, and it made it possible to classify a pre-processed satellite image, as already described in Section 2.1.1. The plugin outputs included both the image that had its pixels classified, and an optional shapefile "describing" the outcome, which is to say, a shapefile having all of the pieces of information concerning the number of pixels for each polygon in each class of the image. The percentage of pixels classified as asbestos within each polygon was optionally reported.

\subsubsection{Plugin User Interface (UI)}

The assumption is based on having the initial image to be classified as something that is organized in a single folder, which includes only the tiles that the image is made of, or the entire image itself.

Being a supervised classification, it is also assumed that the user has a raster with some true points, namely, some buildings' cladding in a definitely known material, which can be used as a classification training set. Each single class is identified by a shapefile, with one different shapefile for each class, thus uniquely identifying a type of cladding that is known in advance. These shapefiles are saved, for convenience, in a single folder containing only such files.

In short, there must be:

- The image to be classified, whether it is single or divided into tiles, kept together in a single folder;

- A folder containing the shapes corresponding to each identified class;

- The image to be used as a training set, where different shapes are represented.

The plugin interface is depicted in Figure 5. The different items have the following meanings:

- Select raster(s) to be classified (folder): by selecting the 'browse' button, it is possible to search the folder that has the image (or images) to be classified.

- Select training shapes (folder): by selecting the 'browse' button, it is possible to search the folder that has shapefiles corresponding to the different classes of the training set.

- Select training raster (.tif): by selecting the 'browse' button, it is possible to search the geo-referenced image (filename extension '.tif') whose pixels conform to the shapefiles set out in point 2.

- Create shape count: by ticking this (which is optional), the plugin creates not only a classified image, but also a shapefile $\left({ }^{*}\right.$.dbf) containing all of the polygons that correspond to each cladding. In this shape, each polygon (building) is provided with the following information: the identification 
code, the total number of pixels that each polygon has, and the pixel number of each identified class in the polygon.

- Create shape percentage: by ticking this (which is optional), the plugin creates not only a classified image, but also a shapefile $\left({ }^{*} . \mathrm{dbf}\right)$ containing all of the polygons that correspond to each cladding. In this shape, each polygon (building) is provided with the following information: the identification code, and the percentage of classified pixels within each polygon for each identified class in that polygon.

- Select the file (.shp) to create a shape count/percentage: whether one of the two previously mentioned options or both of them have been ticked, this box makes it possible to select the shapefile to be used when calculating the required statistics. Generally speaking, this shapefile is the same as the one that is used in the filtering phase of the satellite image.

- Select the output folder: when clicking on 'browse', it is possible to select the folder where the output files will be saved.

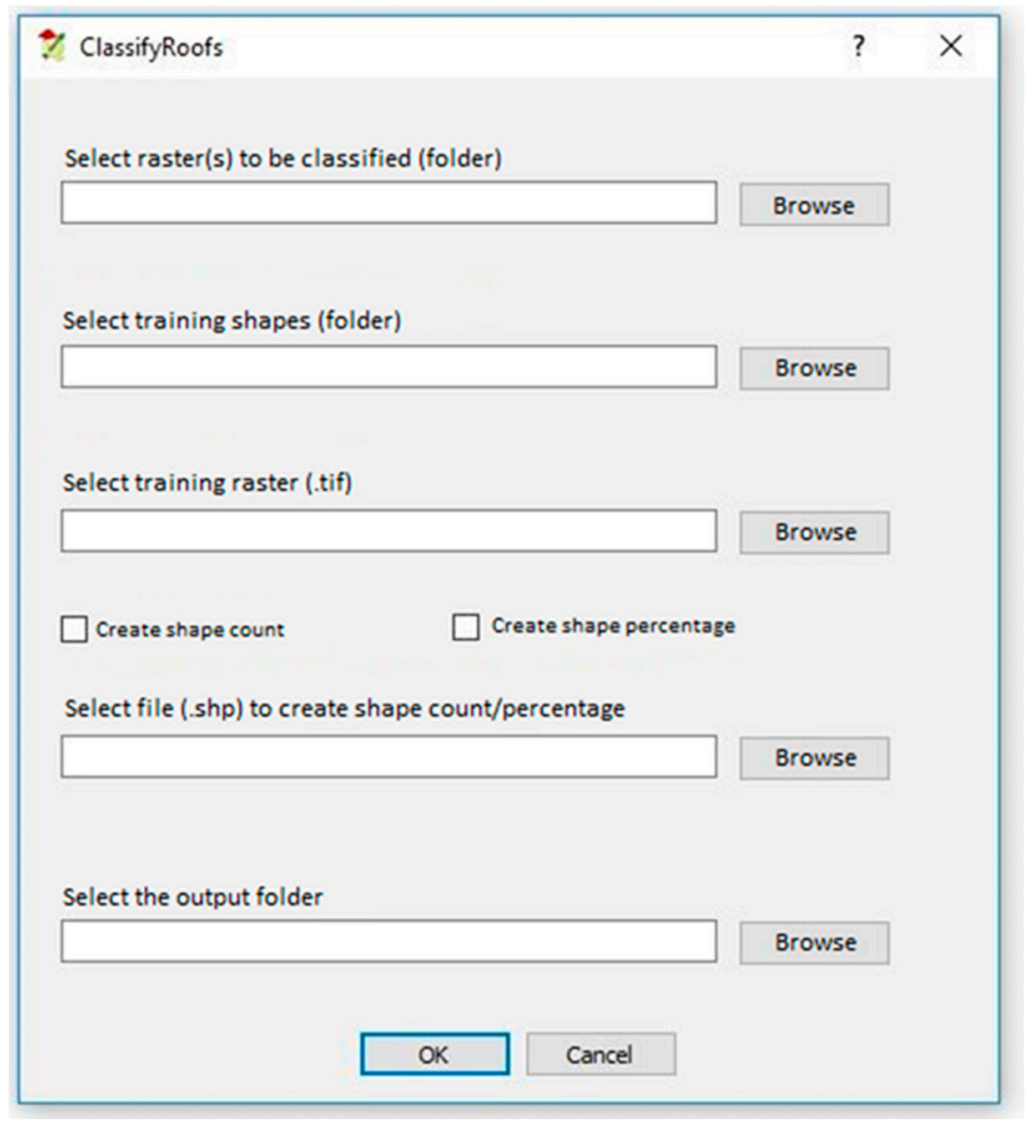

Figure 5. User Interface of the RoofClassify plugin.

2.2.2. Focusing on How the Classification Plugin is Organized

The plugin performs the classification according to the following procedural points:

1. Importing the raster image to be classified (images available in the selected folder as input dataset) into a Python data structure (multi-array), where each and every pixel matches exactly with a cell of the structure, made of eight components (one for each band).

2. Importing the raster image, which represents the selected training set for classification purposes (same as point 1 ).

3. Extracting the training set from the shapefile: a shapefile is defined for each class to be identified, and it includes the polygons matched with the related roofing. As previously explained, each 
and every shape (one shape matching with the asbestos class, one shape for a red roof, and so forth) is saved in a single folder. The algorithm will recursively access this folder, looking for the shapes in order to extract the training set. These data are also imported into a Python multiarray.

4. Training the classifier file: the training and classification algorithm belongs to the "Scikit-learn" Python library. The multiclass "RandomForest" classification function was used in this case, which was more suitable for execution speed. The inputs were: the multiarray raster of the training set (point 2); the multiarray shapes of the training set (point 3).

5. Classification according to the selected function. The output will be a multiarray comprising the classified pixels' values.

6. Reconstructing and saving the correctly geo-referenced image as the starting image for use, beginning with the output provided in point 5 .

Some items were left out of the above description for the sake of brevity, namely, the option of creating the shapefile with all of the information on classification.

\section{Classification Results and Validation}

The outcome that is achieved by applying the plugin to the pre-processed image is an image that has pixels classified according to the different types of roofing. Such an image is correctly geo-referenced according to the source satellite image. Figure 6 is an example of a part of an image with classified pixels.

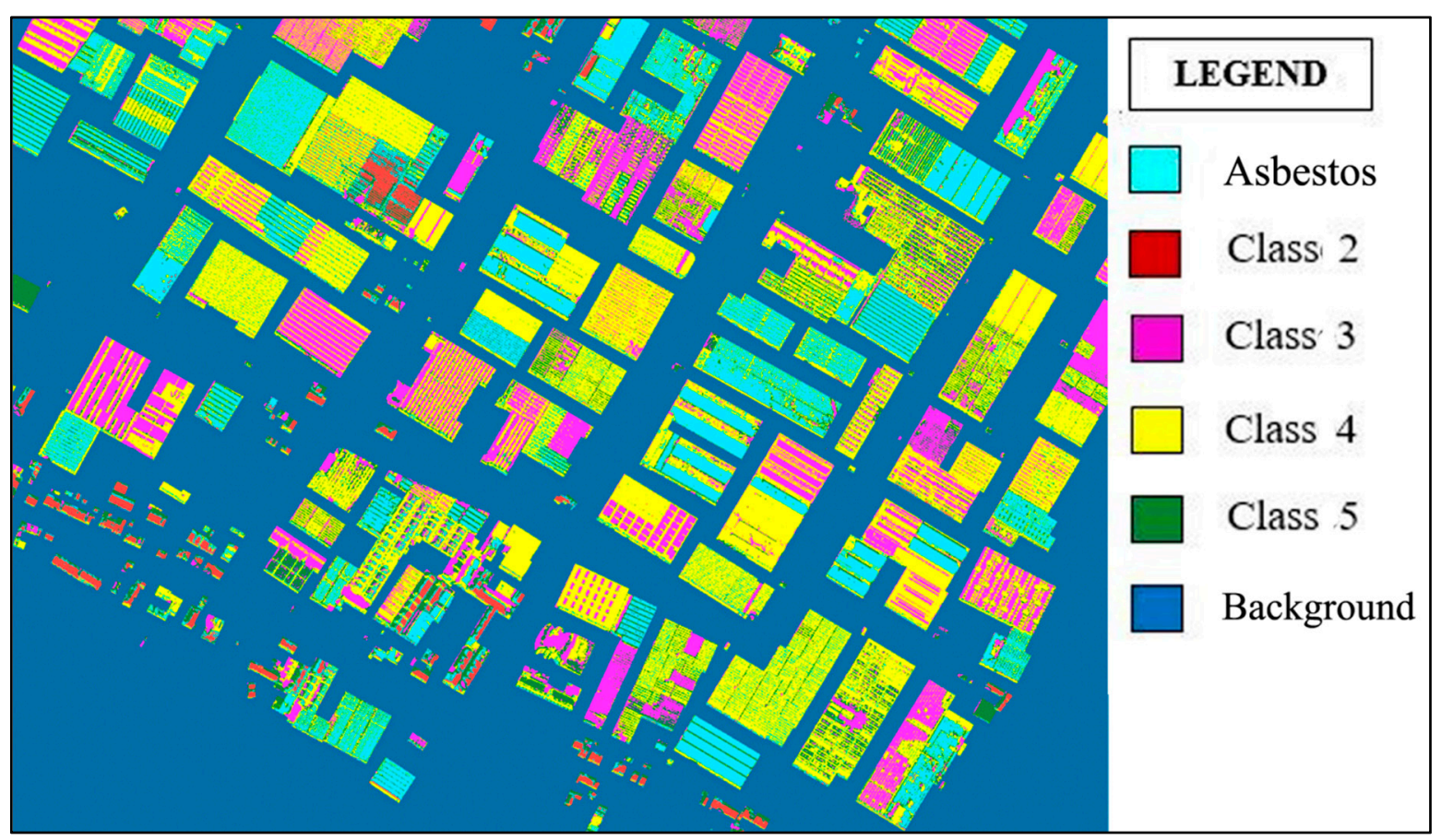

Figure 6. A part of a classified image.

With regard to each building in the input shape file (Shapefile Mask), once the classified image is available, the designed algorithm identifies the total number of pixels in that image, and for each identified class, the total number of pixels belonging to that specific class.

When the classification is complete, the worthiness of such a process needs to be demonstrated, by validating its outcome. However, given the nature of this use, specifically, the lack of sufficient samples of a well-known nature, especially in dealing with asbestos as the class of interest, it becomes more and more difficult to carry out validations with the criteria and techniques that are most common in the literature. 
Therefore, the following actions were performed to verify the worthiness of this classification method:

1. Identifying the building roofing with well-known construction materials as well as their tagging according to the corresponding class.

2. Creating a shapefile for each class, which included the previously tagged roofing types for each file, according to a specific class.

3. Developing a Python script. Provided with input data, which consisted of both the image created from the classification process, and the shapefiles, as described in point 2, this script is capable of providing a confusion matrix as an output.

4. Analyzing the confusion matrix and estimating any possible classification errors.

The first phase is very critical, and it draws the line at the validation process; in fact, the classification process classifies each pixel according to the dominantly present classes, but an ambiguity arises when asbestos and fiber cement classes are concerned. Indeed, there is a problem with correctly allocating the tag "cement" or "asbestos" to pixels belonging to the available images.

The action taken in order to address a similar situation for such surfaces was to use the corresponding "true points" —obviously not the ones selected for the training set-as test buildings for the asbestos class. Other asbestos-free, newly registered, and recently built structures were used as test buildings for the cement class.

The outcomes are described in Table 2, which represents the confusion matrix, or the taking into account of the outcomes of mistaken classification relating to the parts of the image: the first-line cells show the true classes, whereas the first-column cells show the output classes according to the classification tool. There were six true/output classes of this matrix, according to the number of classes that collected roofing and background.

Table 2. Confusion matrix. CA is commission accuracy, OA is omission accuracy.

\begin{tabular}{cccccccc}
\hline & Asbestos & Cement & Black/Shadow & Green & Background & Red & OA (\%) \\
\hline Asbestos & 13,992 & 4036 & 120 & 39 & 17 & 422 & 75.12 \\
Cement & 682 & 9779 & 321 & 252 & 0 & 126 & 87.63 \\
Black/Shadow & 1 & 88 & 706 & 9 & 0 & 1 & 87.70 \\
Green & 368 & 544 & 140 & 2312 & 1 & 173 & 65.35 \\
Background & 0 & 0 & 0 & 0 & 1346 & 0 & 100 \\
Red & 415 & 853 & 486 & 150 & 120 & 13,867 & 87.26 \\
CA (\%) & 90.52 & 63.91 & 39.82 & 83.71 & 90.70 & 95.5 & 81.77 \\
\hline
\end{tabular}

In this regard, if an " $\mathrm{i}$ " index is assigned to the seven rows representing true classes, and a " $\mathrm{j}$ " index is also assigned to the seven output classes, the cell corresponding to the " $\mathrm{i}$ "-indexed row and the " $\mathrm{j}$ "-indexed column shows the number of pixels that are labeled as belonging to the " $\mathrm{j}$ "-indexed class, according to the classification process, whereas in reality they belong to the " $\mathrm{i}$ "-indexed class.

In order to evaluate the quality of the results, the eighth row and the eighth column were added, respectively containing the percentage values of the commission accuracy (CA), namely, the user's accuracy, and the omission accuracy (OA) [30]. Errors of omission represented false negatives. Errors of commission represented false positives. The overall or average accuracy was $81.77 \%$ (reported at the bottom right corner of the matrix). The Kappa index of the classifier was 0.7514 [31].

As evident from the table, many pixels belonging to "cement" and "asbestos" classes were used in this test, with respect to other classes like "green" or "shadow". This choice was motivated by the classification method, which focused on asbestos detection. Therefore, when calculating the process accuracy, greater consideration should have been given to the class to be detected, as well as to the other classes that asbestos could have been misclassified with.

It turns out that the percentage of correction classification that was related to asbestos detection amounted to $75.12 \%$ when analyzing the confusion matrix. 
Another validation was carried out on the basis of the data collected in the Health Information System for Collective Prevention (SISPC) [32], which is a regional database that provides the stake-holders with a tool to manage health activities and data in a uniform way throughout the regional territory. Clean-up plans, as well as removal and disposal procedures of products and artifacts contaminated with asbestos, are presented online, by means of the SISPC. Therefore, this system provided data with the highest "evidentiary" value, which was needed for a complete validation of the classification data. A validation of the SISPC data was carried out by visual examination, where the related depollution location was checked via Google maps, by identifying the roofing that could be consistent with the declared remediation, and by verifying whether the classification, previously carried out on the image before any clean-up operation, had detected asbestos in that area. A selection was made for both residential and industrial buildings, with 11 different addresses. As for classification accuracy, the results on asbestos-classified pixels were better than the outcome obtained with the previously explained validation method. Indeed, the plugin RoofClassify detected $90 \%$ of asbestos roofs. The differences between the validation tests lied in the lower spectral ambiguities of the selected buildings in the second validation procedure.

\section{Discussion}

The QGIS tool described in this paper was conceived for identifying building roofs with asbestos. Roofing classification was performed through the digital processing of images obtained by the WorldView-3 sensor. Such images were pre-processed, so as to make them suitable for subsequent classification [20]. The outcomes, as achieved in an open context, underwent a validation process, which consisted of analyzing the confusion matrix, and subsequently assessing the classification errors. Given that the most difficult task was testing the tool's impact in discerning asbestos and cement, the decision was made to only use structures with well-known roofing materials as the test buildings, according to recent surveys [18-20]. A second validation was carried out on the basis of a verification process, with data being collected at the Health Information System for Collective Prevention (SISPC) [32].

The results obtained from the first validation showed that about $25 \%$ of the asbestos roofing was a false negative. At the same time, a high number of asbestos pixels were classified as cement pixels, because asbestos-reinforced cement often contains only $6 \%$ of asbestos fiber. These observations could lead to the conclusion that a weak performance by the classification method was obtained $[20,30]$.

Improvements in asbestos roofing identification are possible when resorting to object-oriented classification [22]. As previously mentioned, the designed algorithm identifies the total numbers of pixels in that image, and the total number of pixels belonging to each specific class. The plugin can also compose an optional shapefile that describes all of the pieces of information concerning the number of pixels in each class, for each polygon that composes the images. Optionally, the plugin provides the percentage of pixels that are classified as asbestos, within each polygon. This optional shapefile makes it possible to estimate the probability that a building has asbestos roofing. Therefore, it is possible to assign the "asbestos" or "not asbestos" labels to each roof. This procedure should significantly improve classification performance. This last step is left to the user, who can visualize the data that is extracted from the algorithm.

The tool for asbestos identification in the QGIS platform requires a basic grasp of GIS, but the open-source environment makes it easier for both software use and algorithm distribution. A plugin limit is built into the algorithm, which is conceived to only identify asbestos. Strictly speaking, this feature cannot be considered a flaw, nor a limit on flexibility: in fact, the plugin is meant to perform a specific classification of asbestos, and it has been optimized for this individual purpose, making it different from other proprietary software. 


\section{Conclusions}

This paper has focused on an asbestos identification system to give support to the asbestos roofing disposal policies and programs in small geographical areas. The classification tool is based on GIS open source software, and it was used to analyze Prato district satellite images obtained with the WorldView-3 sensor. The QGIS tool has shown quite good performance in identifying asbestos roofing when applying a per-pixel classification. The percentage of asbestos pixels contained in each roof of the analyzed image can also be determined with this tool. This value can be used for improving the classification process. The presented classification methods can be explained in terms of restoring a data sheet, or a list of records on asbestos roofing buildings, and it can provide a low-cost basis for building up a larger knowledge database, where geographical data are integrated with other data from different administrative sources (as, for instance, SISPC).

Author Contributions: Conceptualization, Maurizio Tommasini and Monica Gherardelli; methodology, Maurizio Tommasini; software, Alessandro Bacciottini; validation, Alessandro Bacciottini; formal analysis, Maurizio Tommasini and Monica Gherardelli; data curation, Alessandro Bacciottini; writing—original draft preparation and editing, Monica Gherardelli; supervision, Maurizio Tommasini and Monica Gherardelli.

Funding: This research was funded by the MUNICIPALITY OF PRATO. The APC received no external funding.

Conflicts of Interest: The authors declare no conflict of interest. The funder had no role in the design of the study; in the collection, analyses, or interpretation of data; or in the writing of the manuscript. The funder approved the publication of the results.

\section{References}

1. Bartrip, P.W.J. History of asbestos related disease. Postgrad. Med. J. 2004, 80, 72-76. Available online: https:// pmj.bmj.com/content/postgradmedj/80/940/72.full.pdf (accessed on 1 February 2019).

2. Suzuki, Y.; Yuen, S.R.; Ashley, R. Short, thin asbestos fibers contribute to the development of human malignant mesothelioma: pathological evidence. Int. J. Hyg. Environ. Healt 2005, 208, 201-210. [CrossRef] [PubMed]

3. Szabó, S.; Burai, P.; Kovács, Z.; Szabó, G.; Kerényi, A.; Fazekas, I.; Paládi, M.; Buday, T.; Szabó, G. Testing Algorithms for the Identification of Asbestos Roofing Based on Hyperspectral Data. Environ. Eng. Manag. J. 2014, 143, 2875-2880. [CrossRef]

4. Barrile, V.; Bilotta, G.; Pannuti, F. A Comparison Between Methods-A Specialized Operator, Object Oriented and Pixel-Oriented Image Analysis-To Detect Asbestos Coverages in Building Roofs Using Remotely Sensed Data. In Proceedings of the International Archives of the Photogrammetry, Remote Sensing and Spatial Information Sciences, XXI ISPRS Congress, Beijing, China, 3-11 July 2008; Volume XXXVII, pp. 427-434. Available online: http://www.isprs.org/proceedings/XXXVII/congress/8_pdf/2_WG-VIII-2/ 51.pdf (accessed on 22 January 2019).

5. Bhaskaran, S.; Paramananda, S.; Ramnarayan, M. Per-Pixel and Object-Oriented Classification Methods for Mapping Urban Features Using Ikonos Satellite Data. Appl. Geogr. 2010, 30, 650-665. [CrossRef]

6. Taherzadeh, E.; Shafri, H.Z.M.; Mansor, S.; Ashurov, R. A comparison of hyperspectral data and WorldView-2 images to detect impervious surface. In Proceedings of the 2012 4th Workshop on Hyperspectral Image and Signal Processing (WHISPERS), Shanghai, China, 4-7 June 2012; pp. 1-4. [CrossRef]

7. Ban, Y.; Jacob, A.; Gamba, P. Spaceborne SAR Data for Global Urban Mapping at 30m Resolution Using a Robust Urban Extractor. ISPRS J. Photogramm. Remote Sens. 2015, 103, 28-37. [CrossRef]

8. Samsudin, S.H.; Shafri, H.Z.M.; Hamedianfar, A. Development of Spectral Indices for Roofing Material Condition Status Detection Using Field Spectroscopy and Worldview-3 Data. J. Appl. Remote Sens. 2016, 10, 025021-025038. [CrossRef]

9. Marino, C.M.; Panigada, C.; Busetto, L. Airborne hyperspectral remote sensing applications in urban areas: Asbestos concrete sheeting identification and mapping. In Proceedings of the IEEE/ISPRS Joint Workshop on Remote Sensing and Data Fusion over Urban Area, Rome, Italy, 8-9 November 2001; pp. 7541-7544.

10. Fiumi, L. Evaluation of MIVIS Hyperspectral Data for Mapping Covering Materials. In Proceedings of the IEEE/ISPRS Joint Workshop on Remote Sensing and Data Fusion over Urban Areas, Rome, Italy, 8-9 November 2001; pp. 324-327. 
11. Bassani, C.; Cavalli, R.M.; Cavalcante, F.; Cuomo, V.; Palombo, A.; Pascucci, S.; Pignatti, S. Deterioration Status of Asbestos-Cement Roofing Sheets Assessed by Analyzing Hyperspectral Data. Remote Sens. Environ. 2007, 109, 361-378. [CrossRef]

12. Fiumi, L.; Campopiano, A.; Casciardi, S.; Ramires, D. Method Validation for the Identification of Asbestos-Cement Roofing. Appl. Geomat. 2012, 4, 55-64. [CrossRef]

13. Frassy, F.; Candiani, G.; Maianti, P.; Marchesi, A.; Nodari, F.R.; Rusmini, M.; Albonico, C.; Gianinetto, M. Airborne Remote Sensing for Mapping Asbestos Roofs in Aosta Valley. In Proceedings of the IEEE International Geoscience and Remote Sensing Symposium (IGARSS 2012), Munich, Germany, 22-27 July 2012; pp. 7541-7544.

14. Frassy, F.; Candiani, G.; Rusmini, M.; Maianti, P.; Marchesi, A.; Rota Nodari, F.; Dalla Via, G.; Albonico, C.; Gianinetto, M. Mapping Asbestos-Cement Roofing with Hyperspectral Remote Sensing over a Large Mountain Region of the Italian Western Alps. Sensors 2014, 14, 15900-15913. [CrossRef] [PubMed]

15. Fiumi, L.; Congedo, L.; Meoni, C. Developing Expeditious Methodology for Mapping Asbestos-Cement Roof Coverings over the Territory of Lazio Region. Appl. Geomat. 2014, 6, 37-48. [CrossRef]

16. Cilia, C.; Panigada, C.; Rossini, M.; Candiani, G.; Pepe, M.; Colombo, R. Mapping of Asbestos Cement Roofs and Their Weathering Status Using Hyperspectral Aerial Images. ISPRS Int. J. Geo-Inf. 2015, 4, 928-941. [CrossRef]

17. Pacifici, F. On the Predictive Value of the WorldView3. VNIR and SWIR Spectral Bands. In Proceedings of the IEEE International Geoscience and Remote Sensing Symposium (IGARSS 2016), Beijing, China, 10-15 July 2016; pp. 898-901. [CrossRef]

18. Taherzadeh, E.; Shafri, H.Z.M. Development of a Generic Model for the Detection of Roof Materials Based on an Object-Based Approach Using Worldview-2 Satellite Imagery. Adv. Remote Sens. 2013, 2, 312-321. [CrossRef]

19. Gibril, M.B.A.; Shafri, H.Z.M.; Hamedianfar, A. New semi-automated mapping of asbestos cement roofs using rule-based object-based image analysis and Taguchi optimization technique from WorldView-2 images. Int. J. Remote Sens. 2017, 38, 467-491. [CrossRef]

20. Abriha, D.; Kovács, Z.; Ninsawat, S.; Bertalan, L.; Balázs, B.; Szabó, S. Identification of roofing materials with Discriminant Function Analysis and Random Forest classifiers on pan-sharpened WorldView-2 imagery-A comparison. Hung. Geogr. Bull. 2018, 67, 375-392. [CrossRef]

21. QGIS. Available online: https://qgis.org/en/site/forusers/download.html (accessed on 15 January 2019).

22. Myint, S.W.; Gober, P.; Brazel, A.; Grossman-Clarke, S.; Weng, Q. Per-Pixel vs. Object-Based Classification of Urban Land Cover Extraction Using High Spatial Resolution Imagery. Remote Sens. Environ. 2011, 115, 1145-1161. [CrossRef]

23. Blaschke, T. Object based image analysis for remote sensing. ISPRS J. Photogramm. Remote Sens. 2010, 65, 2-16. [CrossRef]

24. Digital Globe. Available online: https:/ /www.digitalglobe.com/ (accessed on 22 January 2019).

25. Jenson, J.R.; Cowen, D.C. Remote sensing of urban/suburban infrastructure and socio-economic attributes. Photogramm. Eng. Remote Sens. 1999, 65, 611-622.

26. Orfeo Tool-Box. Available online: https://www.orfeo-toolbox.org/ (accessed on 22 January 2019).

27. Kulkarn, A.D.; Lowe, B. Random Forest Algorithm for Land Cover Classification. Int. J. Recent Innov. Trends Comput. Commun. 2016, 4, 58-63.

28. Whitcomb, J.; Moghaddam, M.; McDonald, K.; Kellndorfer, J.; Podest, E. Wetlands Map of Alaska Using L-Band Radar Satellite Imagery. Can. J. Remote Sens. 2009, 35, 54-72. [CrossRef]

29. Tso, B.; Mather, P. Classification Methods for Remotely Sensed Data; Taylor \& Francis: London, UK, 2001; pp. 309-326.

30. Richards, J.A. Classifier performance and map accuracy. Remote Sens. Environ. 1996, 57, 161-166. [CrossRef]

31. Mclver, D.K.; Friedl, M.A. Estimating pixel-scale land cover classification confidence using nonparametric machine learning methods. IEEE Trans. Geosci. Remote Sens. 2001, 39, 1959-1968. [CrossRef]

32. SISPC-Tuscany Region. Available online: http://opendata.prevenzionecollettiva.toscana.it/ (accessed on 15 January 2019).

(C) 2019 by the authors. Licensee MDPI, Basel, Switzerland. This article is an open access article distributed under the terms and conditions of the Creative Commons Attribution (CC BY) license (http:/ / creativecommons.org/licenses/by/4.0/). 